\title{
Variable association of 24-h peripheral and central hemodynamics and stiffness with hypertension- mediated organ damage: the VASOTENS Registry
}

\author{
Stefano Omboni ${ }^{\mathrm{a}, \mathrm{b}}$, Igor Posokhov c, Gianfranco Parati ${ }^{\mathrm{d}, \mathrm{e}}$, Ayana Arystan $^{\mathrm{f}}$, Isabella Tan ${ }^{\mathrm{g}}$, \\ Vitaliy Barkan $^{\mathrm{h}}$, Natalia Bulanova ${ }^{\mathrm{b}}$, Maria Derevyanchenko', Elena Grigorichevaj, \\ Irina Minyukhinak, Giuseppe Mulè', lana Orlovam, Anna Painin ${ }^{n}$, João M. Peixoto Maldonadoº, \\ Telmo Pereira $^{p}$, Carlos G. Ramos-Becerra ${ }^{q}$, Ioan Tilea ${ }^{r}$, Gabriel Waisman ${ }^{\text {s }}$, on behalf \\ of the VASOTENS Registry Study Group
}

\begin{abstract}
Objective: In this analysis of the telehealth-based Vascular health ASsessment Of The hypertENSive patients Registry, we checked how 24-h central and peripheral

hemodynamics compare with hypertension-mediated organ damage (HMOD).

Methods: In 646 hypertensive patients (mean age $52 \pm 16$ years, $54 \%$ males, $65 \%$ treated) we obtained ambulatory brachial and central SBP and pulse pressure (PP), SBP, and PP variability, pulse wave velocity and augmentation index with a validated cuff-based technology. HMOD was defined by an increased left ventricular mass index (cardiac damage, evaluated in 482 patients), an increased intimamedia thickness (vascular damage, $n=368$ ), or a decreased estimated glomerular filtration rate or increased urine albumin excretion (renal damage, $n=388$ ).
\end{abstract}

Results: Ambulatory SBP and PPs were significantly associated with cardiac damage: the largest odds ratio was observed for 24-h central SBP $[1.032(1.012,1.051)$, $P=0.001]$ and PP $[1.042(1.015,1.069), P=0.002]$, the weakest for brachial estimates. The association was less strong for vascular damage with a trend to the superiority of 24-h central $[1.036(0.997,1.076), P=0.070]$ over brachial PP [1.031 (1.000, 1.062), $P=0.052]$. No statistically significant association was observed for renal damage. SBP and PP variabilities, pulse wave velocity and augmentation index were not associated with any form of HMOD. In the multivariate analysis, age was associated with any type of HMOD, whereas central SBP and PP were predictive of an increased risk of cardiac damage.

Conclusion: In hypertensive patients a variable association exists between peripheral and central hemodynamics and various types of HMOD, with the most predictive power being observed for central SBP and PP for cardiac damage.

Keywords: arterial stiffness, augmentation index, blood pressure, blood pressure telemonitoring, central arterial pressure, hypertension, pulse wave velocity, vascular biomarkers

Abbreviations: ABPM, ambulatory blood pressure monitoring; Alx, augmentation index; ARV, average real variability; BP, blood pressure; CAP, central arterial pressure; e-CRF, electronic case report forms; eGFR, estimated glomerular filtration rate; HMOD, hypertensionmediated organ damage; HR, heart rate; IMT, intimamedia thickness; LV, left ventricular; $L V H$, left ventricular hypertrophy; LVM, left ventricular mass; LVMI, left ventricular mass index; PP, pulse pressure; PWA, pulse wave analysis; PWV, pulse wave velocity; VASOTENS, Vascular health ASsessment Of The hypertENSive patients

\section{INTRODUCTION}

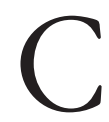
urrently, strong evidence exists that, when measured at rest, central rather than peripheral, hemodynamics are more closely related to hypertension-
Journal of Hypertension 2019, 37:000-000

${ }^{a}$ Clinical Research Unit, Italian Institute of Telemedicine, Varese, Italy, bScientific Research Department of Cardiology, Science and Technology Park for Biomedicine, Sechenov First Moscow State Medical University, Moscow, 'Hemodynamic Laboratory Ltd, Nizhniy Novgorod, Russian Federation, ${ }^{d}$ Istituto Auxologico Italiano, ${ }^{\text {D Department }}$ of Medicine and Surgery, University of Milano-Bicocca, Milan, Italy, 'Department of Functional Diagnostics, Medical Center Hospital of President's Affairs Administration of The Republic of Kazakhstan, Astana, Kazakhstan, 9Department of Biomedical Sciences, Faculty of Medicine and Health Sciences, Macquarie University, Sydney, New South Wales, Australia, h'Diagnostics Department, The Hospital within the Russian Railroad Network, Chita, 'Volgograd State Medical University, Volgograd, 'South Ural State Medical University, Chelyabinsk, ${ }^{k}$ Volga District Medical Center, Nizhniy Novgorod, Russian Federation, 'Unità Operativa di Nefrologia ed Ipertensione, Centro di Riferimento Regionale per I'Ipertensione Arteriosa, Policlinico Paolo Giaccone, Palermo, Italy, mLomonosov Moscow State University Clinic, Moscow, Russian Federation, ${ }^{n}$ Dipartimento di Scienze Mediche e Chirurgiche, Università di Brescia, Medicina 2, Spedali Civili, Brescia, Italy, ${ }^{\circ}$ Clínica da Aveleira, Instituto de Investigação e Formação Cardiovascular, PEscola Superior de Tecnologia da Saúde de Coimbra, Instituto Politécnico de Coimbra, Coimbra, Portugal, ${ }^{9}$ Arterial Stiffness Laboratory, Department of Physiology, University of Guadalajara, Mexico, 'Second Internal Medicine Clinic, Department of Cardiology, County Emergency Clinical Hospital, University of Medicine, Pharmacy, Sciences and Technology, Targu Mures, Romania and 'Istituto Cardiovascular Lezica, Buenos Aires, Argentina

Correspondence to Stefano Omboni, MD, Clinical Research Unit, Italian Institute of Telemedicine, Via Colombera 29, Solbiate Arno, 21048 Varese, Italy. Tel: +39 0331 984176; e-mail: stefano.omboni@iitelemed.org

Received 22 July 2019 Revised 16 September 2019 Accepted 15 October 2019 J Hypertens 37:000-000 Copyright (c) 2019 Wolters Kluwer Health, Inc. All rights reserved.

DOI:10.1097/HJH.0000000000002312 
mediated organ damage (HMOD), though evidence has not always been consistent [1]. In particular, a positive and close relationship has been documented between central arterial pressure (CAP) and left ventricular (LV) mass index (LVMI) and intima-media thickness (IMT), markers of heart and carotid damage, whereas poor or no relation has been shown with impaired renal function [1].

The introduction in recent years of cuff-based technologies able to provide an indirect measurement of central hemodynamics, arterial stiffness and wave reflections over the 24-h through pulse wave analysis (PWA) of oscillometric brachial tracings has the advantage of providing information on the behavior of vascular health in dynamic conditions during the waking hours and notably during the night sleep hours [2]. As it is well known that HMOD is more closely related to ambulatory than to office blood pressure (BP) [3-5] and since some proof exists that this also applies to measurements of CAP $[6,7]$ and to a lesser extent of pulse wave velocity (PWV) [8], routine measurements of ambulatory central hemodynamics may have some potential for a more affordable and detailed assessment of vascular health in daily-life conditions.

The VASOTENS (Vascular health ASsessment Of The hypertENSive patients) Registry [9] is a large web-based repository of ambulatory $\mathrm{BP}$ monitoring (ABPM) recordings obtained with an electronic monitor, which is able to determine CAP, PWV, and augmentation index (AIx) over the 24-h, based on a clinically validated technology of PWA of oscillometric BP measurements [10,11]. Consistency and integrity of data collection are ensured in the study through a telehealth platform, which also provides the engine for the PWA of brachial oscillograms and the extraction of study parameters. As detailed in the publication relative to the study protocol, the study has several objectives [9]. In the current article, we present the results relative to the relationship of peripheral and central hemodynamics, arterial stiffness and wave reflections with HMOD evaluated at the heart, carotid artery and kidney.

\section{METHODS}

\section{Study design and population}

A detailed description of the VASOTENS Registry study protocol may be found elsewhere [9]. The study has an international, multicenter, observational, nonrandomized, prospective design. It is registered with ClinicalTrials.gov at number NCT02577835 and formally endorsed by the Italian and Russian Societies of Hypertension. A total of 24 centers worldwide are involved in the study. For the purpose of the present analysis 14 of these centers provided data: five centers located in Russia, two in Italy, two in Portugal, and one each in Australia, Mexico, Argentina, Romania, and Kazakhstan.

The eligibility criteria for the inclusion of patients into the study were male or female sex; age at least 18 years; a previous diagnosis of arterial hypertension (AH) of any stage or severity or referral to one of the study centers for routine diagnostic evaluation of a suspected hypertension; a need to perform an ABPM for diagnostic purposes. Exclusion criteria were all those conditions preventing from obtaining reliable automated BP measurements with the oscillometric technique (e.g. atrial fibrillation, frequent ectopic beats or second or third-degree atrioventricular blocks, and mid-upper arm circumference $<22 \mathrm{~cm}$ ), and pregnancy. Patients with an arm circumference larger than $32 \mathrm{~cm}$ were not excluded from the study, rather an appropriately sized cuff was used to obtain accurate BP measurements.

The study was performed following the Guidelines for Good Clinical Practice and the Declaration of Helsinki. The protocol was reviewed and approved by the ethics committees of each participating center. All eligible patients willing to participate were fully informed about the study design, purposes, and procedures, and asked to give written informed consent prior to enrolment into the study.

The project did not involve any type of diagnostic evaluation or pharmacological intervention specifically designed for the study and the Investigator was free to manage the patients included in the Registry according to the requirements of clinical practice and current guidelines. However, as guidelines recommend, once enrolled each patient had to be followed-up with visits occurring at regular intervals: ideally every 6 months, and not less than once a year, for a minimum follow-up of 2 years. In the present report data on HMOD collected on the occasion of the enrolment visit were considered and presented.

Data collection was ensured through a certified webbased telehealth platform (THOLOMEUS; Biotechmed Ltd., Somma Lombardo, Varese, Italy) [12]. At each study visit an ABPM was performed and patient's clinical data, such as family history, anthropometric data (height, weight, and waist circumference), smoking and drinking habits, past and current diseases, therapies, office $\mathrm{BP}$, and laboratory tests, including evaluation of HMOD, were collected and entered on the electronic case report form (e-CRF) located on the study website. ABPM recordings were uploaded on the telehealth platform and handled as detailed in the next section.

\section{Office and ambulatory blood pressure measurement}

Conventional (office) BP was measured in duplicate in the seated position at 2-3-min intervals in all patients, before starting the ABPM and subsequently recorded on the e-CRF. Readings were obtained with the same device used for ABPM or with a validated automatic or manual BP measuring device, after $5 \mathrm{~min}$ of rest and under standardized conditions, as recommended by current guidelines. ABPM recordings were performed according to current recommendations [13]. For this purpose, the clinically validated and accurate electronic, automated, upper arm BPLab device (BPLab GmbH, Schwalbach am Taunus, Hessen, Germany) was employed $[10,11,14]$.

The ABPM device was programmed to have intervals between measurements of at least 20 min during the day (providing a minimum of three readings per hour) and at least 30 min during the night (providing a minimum of two readings per hour). The monitoring cuff was placed around the nondominant arm. Patients were free to attend their usual daily activities during ABPM. However, they were required to refrain from strenuous exercise during the monitoring period and to remain motionless during each 
automated BP measurement. They had to complete a diary in which daily activities such as time of sleeping, time of meals, etc. had to be reported together with the time of occurrence of unusual events or poor night sleep quality. The patient had to come back to the outpatient clinic $24 \mathrm{~h}$ after fitting the device and starting the recording to have the monitor removed and the recording uploaded on the webbased telemedicine platform. To this purpose, the ABPM device was plugged to the personal computer through a universal serial bus cable. Then, ABPM data were uploaded and transmitted to the website, where they were analyzed in real-time with the production of an electronic report sent by e-mail to the Investigator and simultaneously published in the user-restricted area of the website. Once the results were obtained from the web-based analysis software, the Investigator checked each recording for compliance with quality criteria (see below for details).

\section{Pulse wave analysis}

The oscillometric BPLab device allows simultaneous measurement of oscillometric brachial BP, arterial stiffness, and central hemodynamics in ambulatory conditions. According to the technique used in this study, vascular biomarkers were obtained by recording pulsatile pressure changes at the brachial artery level during a step-by-step upper arm cuff deflation. When data stored in the device memory were uploaded on the web-based telemedicine platform, the software processed the signal using proprietary mathematical algorithms. These were based on a specifically developed hemodynamic model to get the PWV and transfer function that utilizes a modification in a certain frequency range within the acquired pulse signal to derive the central pressure wave, and thus to assess CAP and AIx. CAP was estimated relative to measured brachial SBP and DBP, as common for other type I devices. A detailed description of the methodology may be found elsewhere $[2,10,11]$. The accuracy of the BPLab device for the assessment of vascular indices has been tested and validated in studies against noninvasive measurements of the same parameters obtained with the Sphygmocor device $[10,11]$.

\section{Assessment of hypertension-mediated organ damage}

HMOD was defined by the presence of LV hypertrophy (LVH), an increased IMT, or renal impairment. All the measurements required for quantification of HMOD were performed at local sites, following current guidelines recommendations. Ultrasound examinations for determination of LVH and IMT were performed by one expert sonographer at each center, whereas blood and urine samples for evaluation of renal function were analyzed at the central laboratories of the single study centers.

Cardiac damage was evaluated through a standard echocardiography examination, following the recommendations of the American Society of Echocardiography [15]. A two-dimensional examination was performed in each participant while lying in a left lateral decubitus position. The interventricular septum thickness, posterior wall thickness, and LV internal diameter were measured at the end of diastole and averaged over three consecutive cardiac cycles. LV mass (LVM) was then calculated according to the formula of Devereux and normalized by the BSA to obtain the LVMI [16]. Cardiac damage was determined by the presence of an LVH, defined by an LVMI greater than $115 \mathrm{~g} / \mathrm{m}^{2}$ in males and greater than $95 \mathrm{~g} / \mathrm{m}^{2}$ in females [17].

Carotid ultrasound examinations were performed to determine the presence of carotid plaque or stenosis. The IMT was measured by carotid ultrasonography at the level of the common carotid artery and averaged over two replicate measurements: an IMT more than $0.9 \mathrm{~mm}$ was deemed compatible with the presence of vascular damage [17].

Renal function assessment was based on the calculation of estimated glomerular filtration rate (eGFR) according to the Cockcroft-Gault equation and/or on the estimation of 24-h albumin excretion, as recommended by current guidelines $[17,18]$. An eGFR less than $60 \mathrm{ml} / \mathrm{min}$ per $1.73 \mathrm{~m}^{2}$ and/ or a urinary albumin excretion at least $30 \mathrm{mg} / 24$-h were considered for renal damage [17].

\section{Statistical analysis}

For the purpose of the present analysis two study populations were identified: first, a full-analysis set, consisting of individuals with a valid estimate of any HMOD and a valid ABPM; and second, one population consisting of patients with all valid estimates of HMOD and valid ABPM. The latter dataset was used for the confirmatory (or sensitivity) analysis.

The analysis of each 24-h ABPM recording was preceded by removal of artifacts according to previously described editing criteria [19]. Recordings were considered valid when at least $70 \%$ of the expected number of readings was obtained, and at least 20 valid awake and seven valid asleep readings were available over the $24-\mathrm{h}$, as recommended by current guidelines [13]. ABPMs matching the aforementioned quality criteria were analyzed to obtain 24-h average of brachial SBP, DBP, and pulse pressure (PP, the difference between SBP and DBP); 24-h average of central SBP, DBP, and PP; 24-h average of PWV; 24-h average of AIx; 24-h brachial and central SBP, DBP, and PP variability quantified by the average real variability (ARV), calculated by averaging the successive absolute differences between adjacent

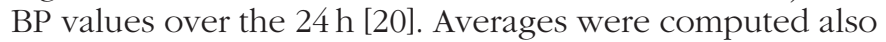
for the daytime and night time subperiods (defined according to the actual night sleep and waking hours). As AIx depends on heart rate (HR), in each individual AIx was normalized to an HR of $75 \mathrm{bpm}$ [21].

Basic demographic and clinical variables were collected for each individual, including a history of $\mathrm{AH}$, dyslipidemia, diabetes mellitus, and cardiovascular disease. Overweight or obesity was defined by a waist circumference at least $94 \mathrm{~cm}$ in males and at least $80 \mathrm{~cm}$ in females for white patients or at least $90 \mathrm{~cm}$ in males and at least $80 \mathrm{~cm}$ in females for Asian and Hispanic patients. When waist circumference was missing a BMI of at least $25 \mathrm{~kg} / \mathrm{m}^{2}$ was used to evaluate overweight or obesity.

Descriptive statistics were provided for all demographic, clinical, and hemodynamic variables by calculating absolute and relative frequencies (categorical variables) and average value $\pm S D$ or $95 \%$ confidence interval (CI) (continuous variables). The analysis was run for subgroups according to the type of HMOD assessment cardiac (LVH absent vs. present); vascular (carotid thickening absent vs. 
present); renal (reduced renal function or albuminuria absent vs. present). In each subgroup differences in hemodynamic indices were assessed by analysis of variance with no adjustment (crude estimate) and after accounting for the center effect and for those factors resulting significantly different between groups in the univariate test (usually age, sex, AH, dyslipidemia, diabetes, cardiovascular disease, and obesity). To address the potential influence within each HMOD group of other types of HMOD, adjustment for the type of HMOD was also entered in the model. In the case of comparisons affecting AIx and ARV an additional adjustment for the mean BP level was also applied.

The association of each SBP and PP estimate, AIx and PWV, with the presence of HMOD was assessed by means of stepwise logistic regression analyses, by calculating odds ratio (OR) and 95\% CI adjusted for confounding factors, as in the analysis of variance test. Due to the collinearity between the different BP indices, these parameters were introduced in each model one at a time. A logistic regression analysis was also performed by introducing in the model at the same time all the peripheral and central BPs, separately for SBP and PP. Data management and analysis were carried out by SPSS for Windows version 25 (IBM Corp., Armonk, NY, USA). A $P$ less than 0.05 was considered as the minimum level of statistical significance.

\section{RESULTS}

\section{Demographic and clinical data}

A total of 646 patients with at least one type of HMOD assessment and with valid 24-h ABPMs were included in this analysis. The average number of valid ambulatory BP readings was 49.6 (range $21-76$ ) during the daytime and 16.4 (range 7-33) during the night-time. Mean patients' age was $51.6 \pm 15.8$ years, $54.0 \%$ of them were males, $72.8 \%$ reported a previous diagnosis of AH $(65.3 \%$ were treated with antihypertensive medications), 44.9\% had dyslipidemia, $10.7 \%$ diabetes, $20.1 \%$ a cardiovascular disease, and $76.0 \%$ were overweight or obese.

Of these 646 patients, $482(74.6 \%)$ had an evaluation of cardiac damage, 368 (57.0\%) of vascular damage and 388 (60.0\%) of renal damage (264 only eGFR, 66 only urine albumin excretion and 58 both). Cardiac damage was detected in 232 of 482 patients (48.1\%), vascular damage in 206 of 368 patients (56.0\%) and renal damage in 61 of 388 patients (15.7\%). Details on demographic and clinical data of the study population according to subgroups of HMOD are reported in Table 1 . These data were homogenous across HMOD subgroups.

\section{Cardiac damage}

In the 482 patients in which echocardiograms were available, LVMI averaged to $132.8 \pm 26.2 \mathrm{~g} / \mathrm{m}^{2}$ in case of $\mathrm{LVH}$, a value which was significantly higher than that measured in patients without LVH $\left(86.3 \pm 16.3 \mathrm{~g} / \mathrm{m}^{2}, \quad P=0.0001\right)$. Adjusted 24-h, daytime and night-time brachial and central SBP and PP values (Fig. 1) and clinic brachial SBP and PP values (Table 2) were significantly higher in patients with than in those without LVH, whereas no statistically significant between-groups difference was observed for DBP. Brachial and central SBP variability, central PP variability,

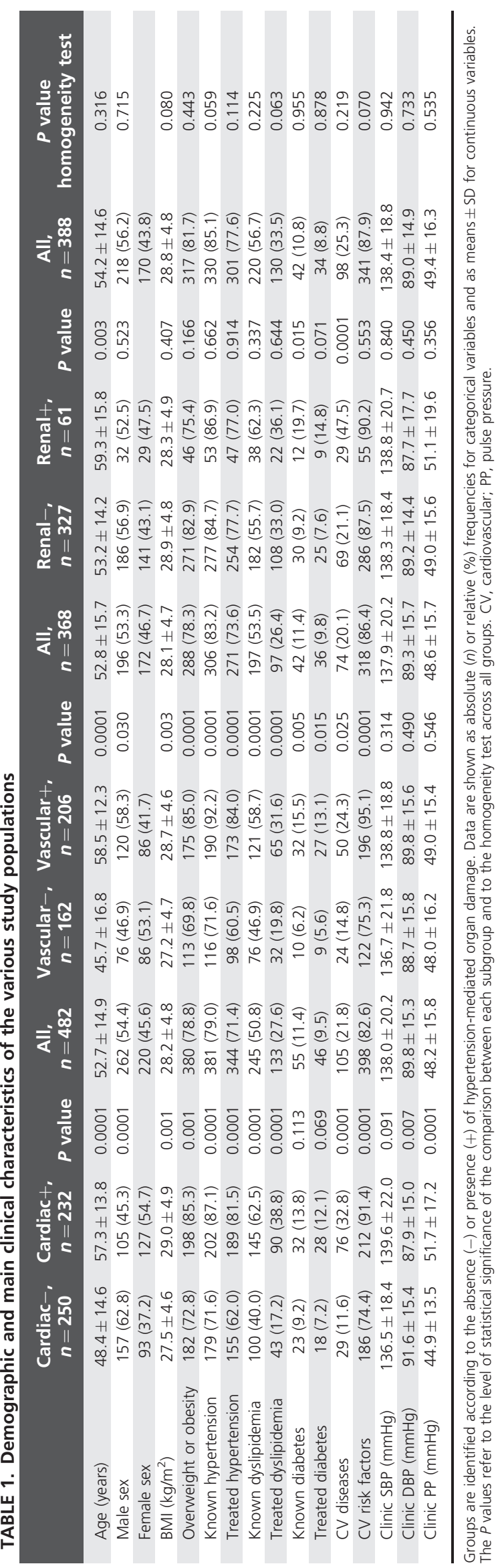


Cardiac damage $(n=482)$
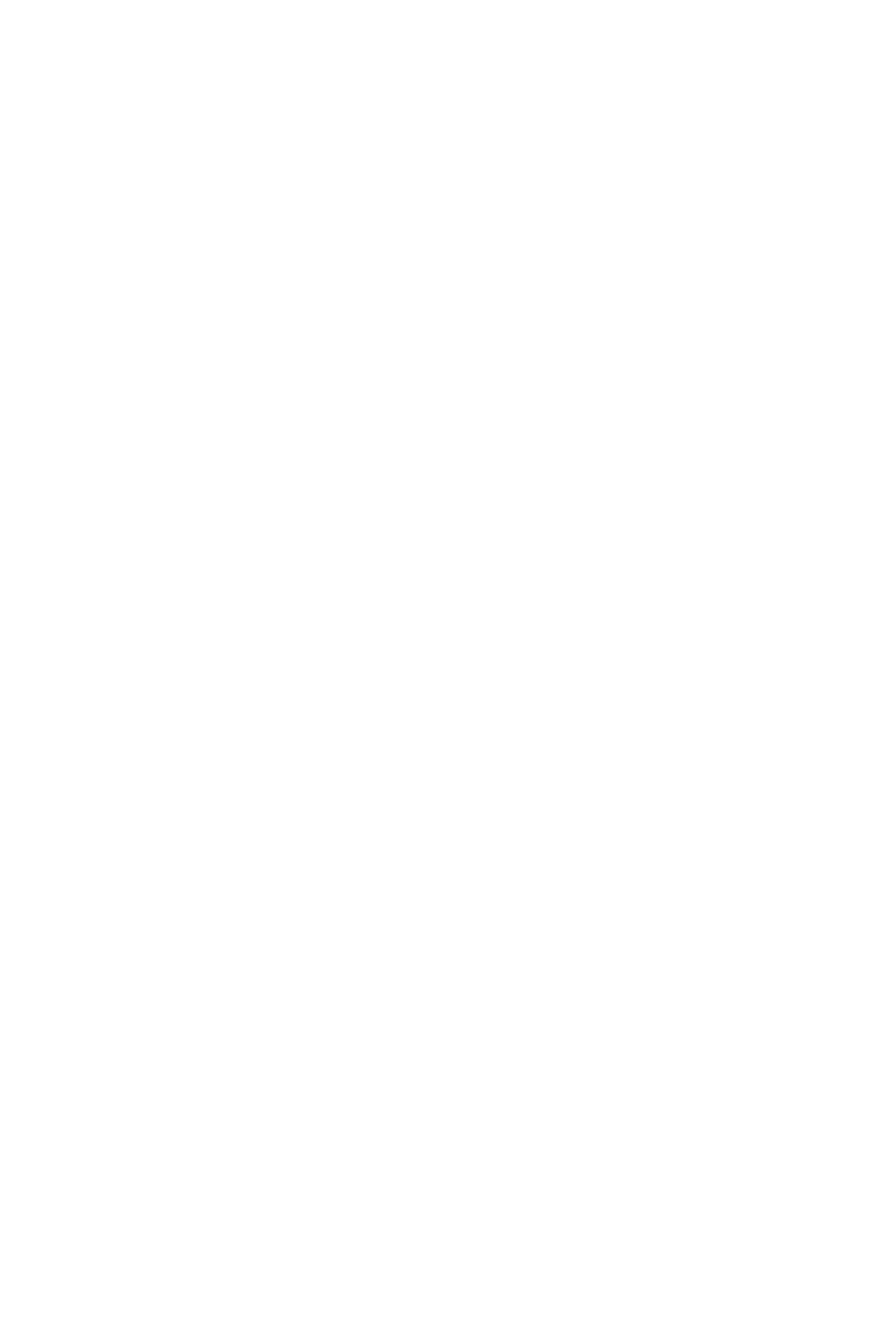









